\title{
Kolmogorov turbulence defeated by Anderson localization for a Bose-Einstein condensate in a Sinai-oscillator trap
}

\author{
Leonardo Ermann, ${ }^{1,2}$ Eduardo Vergini, ${ }^{1}$ and Dima L. Shepelyansky ${ }^{3}$ \\ 1 Departamento de Física, Gerencia de Investigación y Aplicaciones, \\ Comisión Nacional de Energía Atómica. Av. del Libertador 8250, 1429 Buenos Aires, Argentina \\ ${ }^{2}$ CONICET, Godoy Cruz 2290 (C1425FQB) CABA, Argentina \\ ${ }^{3}$ Laboratoire de Physique Théorique, IRSAMC, Université de Toulouse, CNRS, UPS, 31062 Toulouse, France
}

(Dated: March 21, 2017)

\begin{abstract}
We study the dynamics of a Bose-Einstein condensate in a Sinai-oscillator trap under a monochromatic driving force. Such a trap is formed by a harmonic potential and a repulsive disk located in the center vicinity corresponding to the first experiments of condensate formation by Ketterle group in 1995. We argue that the external driving allows to model the regime of weak wave turbulence with the Kolmogorov energy flow from low to high energies. We show that in a certain regime of weak driving and weak nonlinearity such a turbulent energy flow is defeated by the Anderson localization that leads to localization of energy on low energy modes. A critical threshold is determined above which the turbulent flow to high energies becomes possible. We argue that this phenomenon can be studied with ultra cold atoms in magneto-optical traps.
\end{abstract}

PACS numbers: 05.45.Mt, 67.85.Hj, 47.27.-i, 72.15.Rn

The Kolmogorov turbulence [1, 2] is based on a concept of energy flow from large spacial scales, where an energy is pumped by an external force, to small scales where it is absorbed by dissipation. As a result a polynomial energy distribution over wave modes is established which has been obtained first from scaling arguments for hydrodynamics turbulence [1, 2]. Later the theory of weak turbulence, based on diagrammatic techniques and the kinetic equation, demonstrated the emergence of polynomial distributions for various types of weakly interacting nonlinear waves [3 5]. However, this theory is based on a fundamental hypothesis directly stated in the seminal work of Zhakharov and Finonenko: "In the theory of weak turbulence nonlinearity of waves is assumed to be small; this enables us, using the hypothesis of the random nature of the phase of individual waves, to obtain the kinetic equation for the mean square of the wave amplitudes". But in finite systems the dynamical equations for waves do not involve Random Phase Approximation (RPA) and the question of RPA validity, and hence of the whole concept of energy flow from large to small scales, remains open.

Indeed, it is known that in a random media with a fixed potential landscape the phenomenon of Anderson localization [6] breaks a diffusive spreading of probability in space due to quantum interference effects, even if the underline classical dynamics of particles produces an unlimited spreading. At present the Anderson localization has been observed for a large variety of linear waves in various physical systems [7]. A similar phenomenon appears also for quantum systems in a periodically driven $a c$-field with a quantum dynamical localization in energy and number of absorbed photons 8 12]. This dynamical localization in energy has been observed in experiments with Rydberg atoms in a microwave field [12, 13] and cold atoms in driven optical lattices [14, 15]. Thus in the localized phase the periodic driving is not able to pump energy to a system even if the classical dynamics is chaotic with a diffusive spreading in energy.

Of course, the Anderson localization takes place for linear waves. The question about its robustness in respect to a weak nonlinearity attracted recently a significant interest of nonlinear science community [16-20] with the first experiments performed in nonlinear media and optical lattices [21, 22]. These studies show that below a certain threshold the Anderson localization remains robust in respect to a weak nonlinearity while above the threshold a subdiffusive spearing over the whole system size takes place. However, the studies are done for conservative systems without external energy pumping. The numerical simulations for a simple model of kicked nonlinear Schrödinger equation on a ring gave indications that an energy flow to high energies is stopped by the Anderson localization for a weak nonlinearity 23] but such a model is rather far from real experimental possibilities with nonlinear media or cold atoms.

In this Letter we consider a realistic system of BoseEinstein condensate (BEC) of cold atoms captured in a Sinai-oscillator trap under a monochromatic force. In fact this system in three dimensions (3D) had been used for a pioneering realization of BEC reported in 24] (see also [25, 26]). It represents a harmonic trap with a repulsive potential in a vicinity of the trap center created by a laser beam. The repulsive potential can be well approximated by a rigid disk which creates scattering of atoms and instability of their classical dynamics. In two dimensions (2D) with a harmonic potential replaced by rigid rectangular walls the systems represents the well-known Sinai billiard where the mathematical results guaranty that the whole system phase space is chaotic with a pos- 
itive Kolmogorov entropy 27. Recently is was shown that the classical phase space remains practically fully chaotic if the rigid walls are replaced by a harmonic potential which is much more suitable for BEC experiments 28 . The corresponding quantum system is characterized by the level spacing statistics of random matrix theory 29] satisfying the Bohigas-Giannoni-Schmit conjecture [30] and thus belonging to the systems of quantum chaos 31 .

The effects of nonlinearity for BEC evolution in a Sinaioscillator trap has been studied in 28] in the frame of the Gross-Pitaevskii equation (GPE) [32]. It was shown 28 that at weak nonlinearity the dynamics of linear modes remains quasi-integrable while above a certain threshold there is onset of dynamical thermalization leading to the usual Bose-Einstein distribution 33 over energies of linear eigenmodes. Even if being chaotic this system has energy conservation and there is no energy flow to high energy modes. However, a monochromatic driving force breaks the energy conservation leading for a classical dynamics to a diffusive energy growth and probability transfer to high energy modes typical for the Kolmogorov turbulence. Here we show that there is a regime where such an energy transfer to waves with high wave vectors is suppressed by the dynamical localization being similar to the Anderson localization in disordered solids.

We note that the Kolmogorov turbulence for BEC in $2 \mathrm{D}$ rectangular and $3 \mathrm{D}$ cubic billiards has been studied numerically in 34, 35. However, the integrable shape of these billiards does not allow to realize a generic case of random matrix spectrum of linear modes typical for our billiard belonging to the class of quantum chaos systems 31 .

For our model the classical dynamics and quantum evolution in absence of interactions are described by the Hamiltonian

$\hat{H}_{0}=\left({\hat{p_{x}}}^{2}+{\hat{p_{y}}}^{2}\right) / 2 m+m\left(\omega_{x}^{2} \hat{x}^{2}+\omega_{y}^{2} \hat{y}^{2}\right) / 2+V_{d}(\hat{x}, \hat{y})+f \hat{x} \sin \omega$

Here the first two terms describe 2D oscillator with frequencies $\omega_{x}, \omega_{y}$, the third term represents the potential of rigid disk of radius $r_{d}$ and the last term gives a driven monochromatic field of amplitude $f$. Here we fixed the mass $m=1$, frequencies $\omega_{x}=1, \omega_{y}=\sqrt{2}, \omega=(1+\sqrt{5})$ and disk radius $r_{d}=1$. The disk center is placed at $\left(x_{d}, y_{d}\right)=(-1 / 2,-1 / 2)$ so that the disk bangs a hole in a center vicinity as it was the case in the experiments 24. In the quantum case we have the usual commutator relations $\left[\hat{p_{x}} \hat{x}\right]=\left[\hat{p_{y}} \hat{y}\right]=-i \hbar$ with $\hbar=1$ for dimensional units.

The BEC evolution in the Sinai oscillator trap is described by the GPE, which reads:

$$
i \hbar \frac{\partial \psi(x, y, t)}{\partial t}=\hat{H}_{0} \psi(x, y, t)+\beta|\psi(x, y, t)|^{2} \psi(x, y, t),
$$

where $\beta$ describes nonlinear interactions for BEC. Here we use the same Sinai oscillator parameters as in [28] with

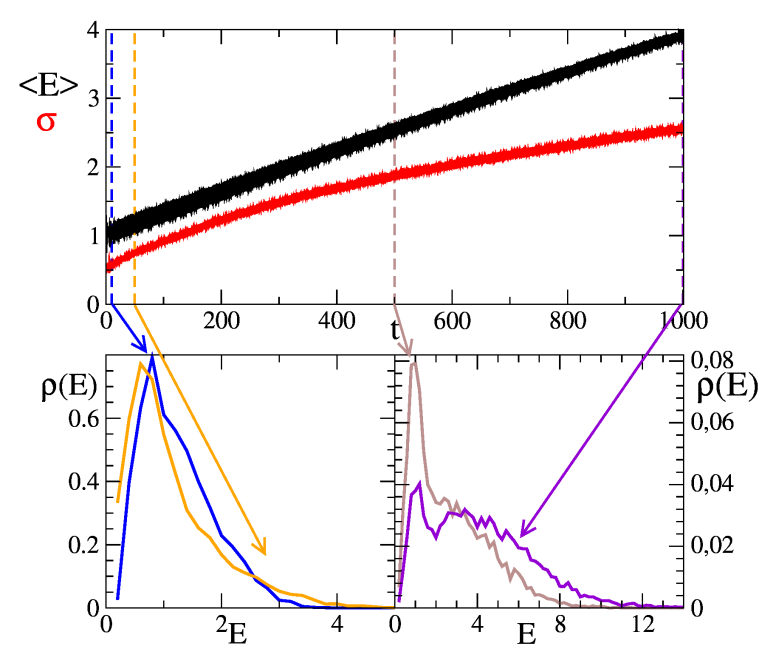

FIG. 1: (Color online) Classical time evolution of average energy $\langle E\rangle$ and its standard deviation $\sigma$ for $f=0.4$. The data are obtained from $10^{4}$ trajectories with random initial conditions at $\langle E\rangle=1$ and $\sigma=0.5$. Top panel: $\langle E(t)\rangle$ and $\sigma(t)$ are shown by black and red/gray curves respectively. Bottom panels show probability distribution of trajectories $\rho(E, t)$ for (a) $t=10,50$ (blue/black, orange/gray curves) and (b) $t=500,1000$ (yellow/gray, violet/black curves). Vertical dashed lines in main panels mark snapshot times corresponding to bottom panels.

normalization $\int|\psi|^{2} d x d y=1$. The numerical integration of (2) is done in the same way as in [11, 28, with a Trotter time step $(\Delta t=0.005)$ evolution for noninteracting part of $\hat{H}_{0}$ followed by the nonlinear term contribution.

The results for energy $E$ growth with time for classical dynamics (1) are shown in Fig. 1. The energy $E$ and its dispersion $\sigma$ are steadily growing with time. We expect that at large times the energy increases diffusively with a rate $(\Delta E)^{2} / t=D \approx C f^{2} \omega_{x}^{2} r_{d} \sqrt{E} / \omega^{2}$ assuming that $\omega_{x} \sim \omega_{y}$ and $\omega>\omega_{x}$. The data of Fig. 1 give us $C \approx 0.5$ at $t=10^{3}$.

We note that the estimate for $D$ comes from the fact that an oscillating velocity component $v_{\text {osc }}=f \cos (\omega t) / \omega$ gives a velocity change at disk collision (like with oscillating wall) $\Delta v_{x}=2 v_{\text {osc }}$ and an energy change $\Delta E \approx$ $v_{x} \Delta v_{x}$ so that the diffusion is $D \sim(\Delta E)^{2} / t_{c}$ where an average time between collisions $t_{c}$ is defined from the ergodicity relation $\Delta t_{c} / t_{c} \sim r_{d}^{2} \omega_{x}^{2} / E$ of ratio of disk area and area of chaotic motion at energy $E$, where $\Delta t_{c} \sim r_{d} / E^{1 / 2}$; thus at large times $E \propto t^{2 / 3}$. The fit for $E \sim \sigma \propto t^{\alpha}$ in Fig. 1 gives $\alpha=0.98 \pm 0.06$ (for $E$ ) and $0.58 \pm 0.08$ (for $\sigma$ ) being comparable to the theoretical value $\alpha=2 / 3$. We attributed a deviation from theory to not sufficiently large amplitude of motion $\sqrt{2 E} / \omega_{x}$ required for $t_{c}$ expression at reached energies.

We also introduce cells of finite energy size $\delta E$ and determine the probability distribution $\rho_{k}$ over $k$ energy cells counting a relative number of trajectories inside each cell. The results of Fig. 1 show that the width of proba- 


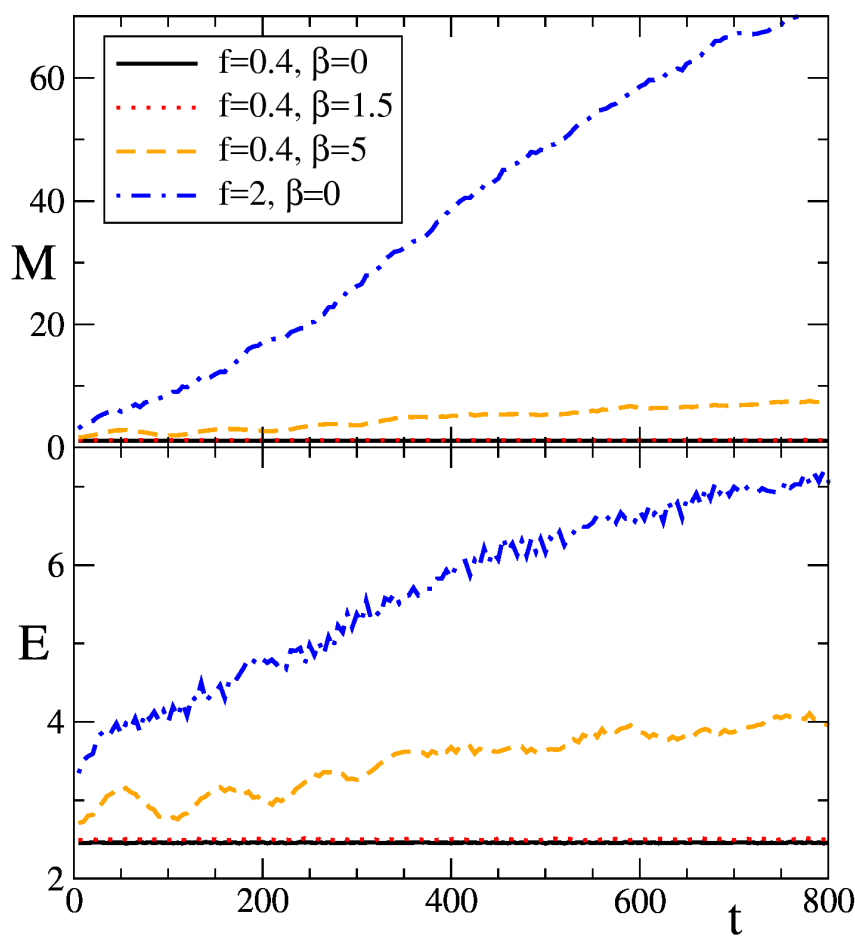

FIG. 2: (Color online) Time evolution of $M$ (top panel) and energy $E$ (bottom panel) for GPE (2) averaged over time intervals $\Delta t=1$. The initial state is the ground state of $(2)$ at $\beta=0, f=0$ (see Fig.5a in [28]). Both panels show the cases of $f=0.4, \beta=0$ (black solid lines), $f=0.4, \beta=1.5$ (red/gray dotted lines), $f=0.4, \beta=5$ (orange/gray dashed lines), $f=2, \beta=0$ (blue/gray dot-dashed lines),

bility distribution $\rho(E)$ in energy is growing in time corresponding to increase of $E$.

The situation is drastically different in the quantum case at $\beta=0$. Here, at small $f$, the dynamical localization leads to a complete suppression of energy $E$ and average mode number $M=\sum_{k} k \rho_{k}$ growth with their restricted oscillations in time (see Fig. 2). The probability distribution $\rho_{k}$ over eigenstates $\psi_{k}$ with eigenenergies $E_{k}$ of (1) (for stationary case $f=0$ ) is shown in Fig. 3 . For small $f<f_{c}$, on average there is a clear exponential decay of probability $\rho_{k} \propto \exp \left(-2 E_{k} / \omega \ell_{\phi}\right)$ with a number of absorbed photons $N_{\phi}=E_{k} / \omega$ and a photonic localization length $\ell_{\phi}$. Such a localization decay is similar to those discussed for atoms [12, 13] and quantum dots [1] in a microwave field. However, above a certain $f_{c}$, e.g. at $f=2 ; 3$, we obtain delocalized probabilities $\rho_{k}$ with a flat plateau distribution at high energies.

According to the theory of dynamical localization described in [11, 12, 36] we have $\ell_{\phi} \approx 2 \pi\left(D / \omega^{2}\right) \rho_{c}$ where $\rho_{c}=d k / d E_{k}$ is the density of $E_{k}$ states. According to [28] we have $k \approx E^{2} / 2 \sqrt{2}$ and $\rho_{c} \approx E / \sqrt{2}$. With the above expression for the classical diffusion in energy $D$ we obtain $\ell_{\phi} \approx 2 f^{2} \omega_{x}^{2} E^{3 / 2} / \omega^{4}$. Similar to the quantum chaos model 36] we have $\ell_{\phi}$ significantly growing with
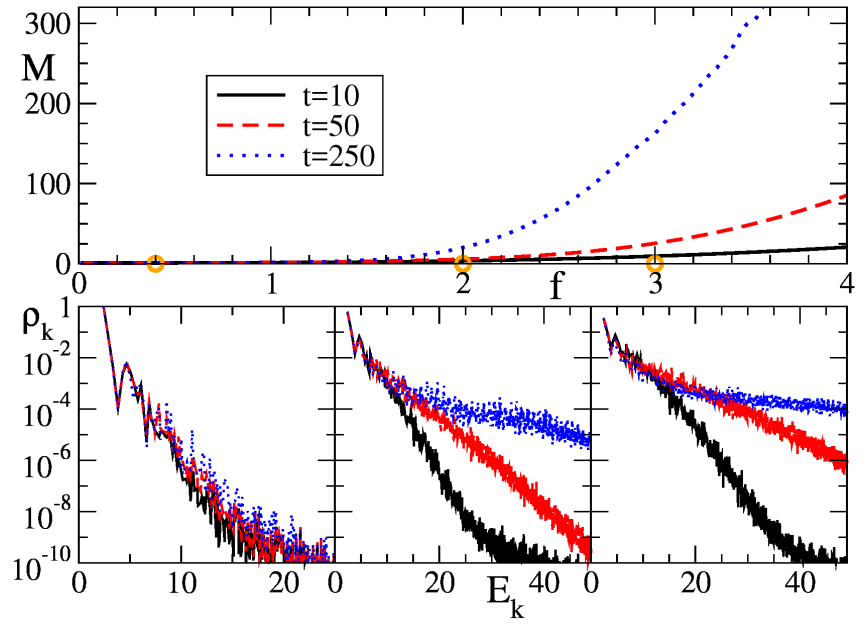

FIG. 3: (Color online) Top panel shows $M$ as a function of driven force $f$ for linear case $(\beta=0)$. Bottom panels show probability distribution $\rho_{k}$, averaged over time interval $\Delta t=5$, as a function of eigenenergies $E_{k}$ with $t=10$ in black solid lines, $t=50 \mathrm{in} \mathrm{red/gray} \mathrm{dashed} \mathrm{lines,} \mathrm{and} t=250$ in blue/gray dotted lines; Left, center and right bottom panels show the cases of $f=0.4,2,3$ respectively (highlighted with orange/gray circles in top panel).

the number of absorbed photons $N_{\phi}$ so that the delocalization of quantum chaos takes place at $\ell_{\phi}>N_{\phi}$. As in 36 this leads to a delocalization above a certain border $f>f_{c}$ with a flat probability distribution on high energies as it is seen in Fig. 3. This gives the delocalization border for quantum states: $f_{c} r_{d} / \hbar \omega_{x} \approx 0.7\left(\omega / \omega_{x}\right)^{3 / 2} \approx 4$ for the initial ground state at $E \approx \hbar \omega_{x}=1$ and $\omega \approx 3.2$. The data for $M$ in Fig. 3 give the critical value $f_{c} \approx 1.5$ being somewhat smaller than the value given by the above estimate. We attribute this difference to the fact that the above estimate for $D$, and hence for $\ell_{\phi}$, is valid in the limit of large spacial oscillations being larger than $r_{d}$. The delocalization transition at $f>f_{c}$ is similar to the Anderson transition, or metal-insulator transition, in disordered systems [6, 7].

The results for $\beta>0$ are presented in Figs. 2, 4. For $f=0.4$, when the steady-state probability is well localized at $\beta=0$, they clearly show that at $\beta=1.5$ there is no growth of energy $E$ and mode number $M$. Thus there is no energy flow to high energies and the Anderson localization remains robust for weak nonlineary perturbation. This is also well confirmed by a stable in time probability distribution over energies $E_{k}$ shown in Fig. 4 (left panel). For larger nonlinearity $\beta=5$ and $f=0.4$ there appears a growth of $M, E$ with time (Fig. 2). At larger $f=1$ and $\beta=5$ there is emergence of energy flow to high energies and increasing probability $\rho_{k}$ at high energies $E_{k}$ (Fig. 4 right panel).

The global dependence of average mode number $M$ on driving amplitude $f$ and nonlinearity $\beta$ is shown in Fig. 5. We see that there is a stability region of small $f, \beta$ 


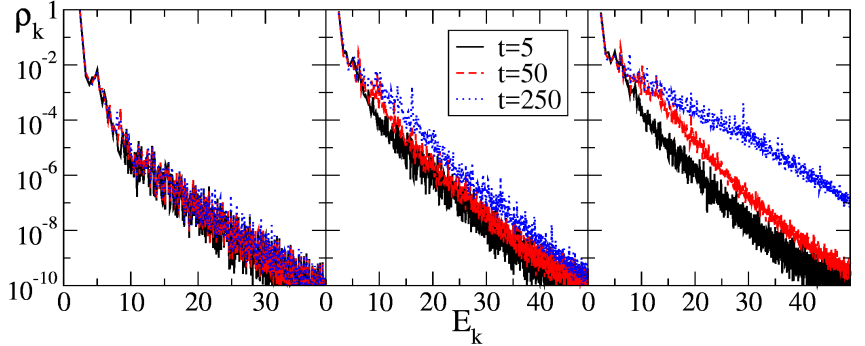

FIG. 4: (Color online) Same as in bottom panels of Fig. 3 for $f=0.3, \beta=1.5$ (left panel), $f=0.5, \beta=5$ (center panel) $f=1, \beta=5$ (right panel)

values where the values $M$ remain small even at large times. This region corresponds to the localized insulator phase (I), from the view point of Anderson localization, of quasi-integrable (or laminar) phase from the view point of nonlinear dinamics (or turbulence). Outside of this region we have large values of number of populated states $M$ so that this regime corresponds to the delocalized metallic or turbulence phase (M-TB). According to the obtained results we conclude that this quasi-stable (or insulator) regime $\left(f<f_{c}, \beta<\beta_{c}\right)$ (see Fig. 5 ) is approximately described by the relation

$$
f_{c} r_{d} / \hbar \omega_{x} \approx 1.5\left(1-\beta_{c} /\left(6 \hbar \omega_{x} r_{d}^{2}\right)\right)
$$

assuming that $\omega_{x} \sim \omega_{y} \sim \omega$. Inside the I-region the turbulent Kolmogorov flow of energy to high modes is suppressed by the Anderson localization. At small nonliniarity $\beta$ we expect a validity of the KolmogorovArnold-Moser theory (KAM) 37, 38, leading to a quasiintegrable dynamics and trapping of energy on large length modes. At the same time we should note that the mathematical prove of KAM for nonlinear perturbation of pure-point spectrum of Anderson localization and the GPE (2) still remains an open challenge [18, 39, 40.

Outside of the stability region (3) a microwave driving transfers the energy flow from low to high energy modes generating the Kolmogorov energy flow. We expect that the energy dissipation and high modes leads to the the Kolmogorov spectrum of energy distribution [4, 5] over modes. Our results show that the RPA is definitely not valid and that, at small amplitudes of a monochromatic driving and small nonlinearity, the Kolmogorov turbulent flow to high modes is defeated by the Anderson localization and the KAM integrability. The transition from KAM phase to turbulence phase corresponds to the insulator-metal transition in disordered systems with the energy axis corresponding to the spatial distance respectively. The KAM or insulator phase corresponds to a usual observation that a small wind (small $f$ amplitude) is not able to generate turbulent waves.

The experimental realization of our system with BEC in a magneto-optical trap corresponds to the experimental conditions described in 24. A monochromatic per-

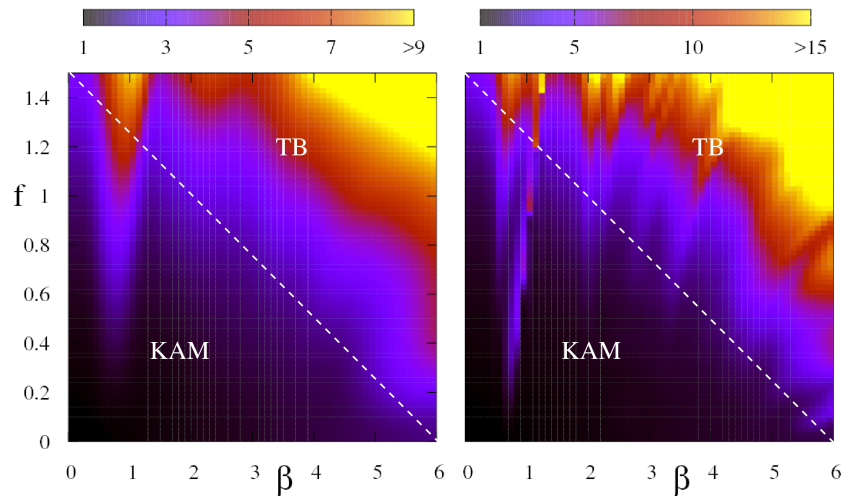

FIG. 5: (Color online) Number of modes $M$ is shown by color/grayness in the plane of parameters $f$ and $\beta$ (average is done in the time intervals $100 \leq t \leq 150$ and $250 \leq t \leq 300$ in left and right panel respectively. The approximate separation of KAM or insulator phase (KAM) and delocalized turbulent or metallic phase (TB) is shown by the white line (3).

turbation can be created by oscillations of the center of harmonic potential or effectively by oscillations of the disk position created by the laser beam. We note that the experimental investigations of turbulent cascades in quantum gases become now possible [41] as well as a thermometry of energy distribution in ultra cold atom ensembles 42. Thus we hope that the interesting fundamental aspects of nonlinear dynamics and weak turbulence will be tested with cold atom experiments.

This work was supported in part by the Pogramme Investissements d'Avenir ANR-11-IDEX-0002-02, reference ANR-10-LABX-0037-NEXT (project THETRACOM).

[1] A.N. Kolmogorov, The local structure of turbulence in an incompressible liquid for very large Reynolds numbers, Dokl. Akad. Nauk SSSR 30, 299 (1941); Dissipation of energy in the locally isotropic turbulence, 32, 19 (1941) [in Russian] (English trans. Proc. R. Soc. Ser. A 434, 19 (1991); 434, 15 (1991)).

[2] A.M. Obukhov, On energy distribution in the spectrum of a turbulent flow, Izv. AN SSSR Ser. Geogr. Geofiz., 5(4-5), 453 (1941) [in Russian].

[3] V.E. Zakharov and N.N. Filonenko, Weak turbulence of capillary waves, J. Appl. Mech. Tech. Phys. 8 (5), 37 (1967).

[4] V.E. Zhakharov, V. S. L'vov and G. Falkovich, Kolmogorov spectra of turbulence, Springer-Verlag, Berlin (1992)

[5] S. Nazarenko, Wave turbulence, Springer-Verlag, Berlin (2011).

[6] P.W. Anderson, Absence of diffusion in certain random lattices, Phys. Rev. 109, 1492 (1958).

[7] E. Akkermans and G. Montambaux, Mesoscopic physics of electrons and photons, Cambridge Univ. Press, Cambridge (2007). 
[8] B.V. Chirikov, F.M. Izrailev and D.L. Shepelyansky, Dynamical stochasticity in classical and quantum mechanics, Sov. Sci. Rev. C - Math. Phys. Rev. (Ed. S.P. Novikov) 2, 209 (1981).

[9] S. Fishman, D.R. Grempel and R.E. Prange, Chaos, quantum recurrences and Anderson localization, Phys. Rev. Lett. 49, 509 (1982).

[10] B.V. Chirikov, F.M. Izrailev and D.L. Shepelyansky, Quantum chaos: localization vs, ergodicity, Physica D 33, 77 (1988).

[11] T. Prosen and D.L. Shepelyansky, Microwave control of transport through a chaotic mesoscopic dot, Eur. Phys. J. B 46, 515 (2005).

[12] D. Shepelyansky, Microwave ionization of hydrogen atoms, Scholarpedia 7(1), 9795 (2012).

[13] P.M. Koch and K.H.A. van Leeuwen, The importance of resonances in microwave ionization of excited hydrogen atoms, Phys. Rep. 256, 289 (1995).

[14] F.L. Moore, J.C. Robinson, C.F. Bharucha, B. Sundaram and M.G. Raizen, Atom optics realization of the quantum $\delta$-kicked rotor, Phys. Rev. Lett. 75, 4598 (1995).

[15] J. Chabe, G. Lemarie, B. Gremaud, D. Delande, P. Szriftgiser and J.C. Garreau, Experimental observation of the Anderson metal-insulator transition with atomic matter waves, Phys. Rev. Lett. 101, 255702 (2008).

[16] D.L. Shepelyansky, Delocalization of quantum chaos by weak nonlinearity, Phys. Rev. Lett. 70, 1787 (1993).

[17] A.S. Pikovsky, D.L. Shepelyansky, Destruction of Anderson localization by a weak nonlinearity, Phys. Rev. Lett. 100, 094101 (2008).

[18] S. Fishman, Y. Krivolapov and A. Soffer, The nonlinear Schrödinger equation with a random potential: results and puzzles, Nonlinearity 25, R53 (2012).

[19] L. Ermann, and D.L. Shepelyansky, Quantum Gibbs distribution from dynamical thermalization in classical nonlinear lattices, New J. Phys. 15, 12304 (2013).

[20] T.V. Laptyeva, M.V. Ivanchenko and S. Flach, Nonlinear lattice waves in heterogeneous media, J. Phys. A: Math. Theor. 47, 493001 (2014).

[21] T. Schwartz, G. Bartal, S. Fishman and M. Segev, Transport and Anderson localization in disordered twodimensional photonic lattices, Nature 446, 52 (2007).

[22] E. Lucioni, B. Deissler, L. Tanzi, G. Roati, M. Zaccanti, M. Modugno, M. Larcher, F. Dalfovo, M. Inguscio and G. Modugno, Observation of subdiffusion in a disordered interacting system, Phys. Rev. Lett. 106, 230403 (2011).

[23] D.L. Shepelyansky, Kolmogorov turbulence, Anderson localization and KAM integrability, Eur. Phys. J. B 85, 199 (2012).

[24] K.B. Davis, M.-O. Mewes, M.R. Andrews, N.J. van Druten, D.S. Durfee, D.M. Kurn, and W. Ketterle, BoseEinstein condensation in a gas of sodium atoms, Phys. Rev. Lett. 75, 3969 (1995).
[25] J.A. Anglin, and W. Ketterle, Bose-Einstein condensation of atomic gases, Nature 416, 211 (2002).

[26] W. Ketterle, Nobel lecture: When atoms behave as waves: Bose-Einstein condensation and the atom laser, Rev. Mod. Phys. 74, 1131 (2002).

[27] Ya.G. Sinai, Dynamical systems with elastic reflections. Ergodic properties of dispersing billiards, Uspekhi Mat. Nauk 25(2), 141 (1970) [English trans.: Russian Math. Surveys 25(2), 137 (1970) ].

[28] L. Ermann, E. Vergini and D.L. Shepelyansky, Dynamics and thermalization a Bose-Einstein condensate in a Sinai-oscillator trap, Phys. Rev. A 94, 013618 (2016).

[29] E. Wigner, Random matrices in physics, SIAM Review 9(1), 1 (1967).

[30] O. Bohigas, M.J. Giannoni, and C. Schmit, Characterization of chaotic quantum spectra and universality of level fluctuation laws, Phys. Rev. Lett. 52, 1 (1984).

[31] F. Haake, Quantum signatures of chaos, Springer, Berlin (2010).

[32] L. Pitaevskii, and S. Stringari, Bose-Einstein condensation, Oxford Univ. Press (2003).

[33] L.D. Landau and E.M. Lifshitz, Statistical physics, Wiley, New York(1976).

[34] S. Nazarenko, M. Onorato, and D. Proment, BoseEinstein condensation and Berezinskii-Thouless transition in the two-dimensional nonlinear Schrödinger model, Phys. Rev. A 90, 013624 (2014).

[35] K. Fujimoto, and M. Tsubota, Bogoliubov-wave turbulence in Bose-Einstein condensates, Phys. Rev. A 91, 053620 (2015).

[36] F. Benvenuto, G. Casati, I. Guarneri and D.L. Shepelyansky, A quantum transition from localized to extended states in a classically chaotic system, Z. Phys. B - Cond. Mat. 84, 159 (1991).

[37] B.V. Chirikov, A universal instability of manydimensional oscillator systems, Phys. Rep. 52, 263 (1979).

[38] A.J.Lichtenberg, M.A.Lieberman, Regular and chaotic dynamics, Springer, Berlin (1992).

[39] J. Bourgain and W.-M. Wang, Quasi-periodic solutions of nonlinear random Schrdinger equations, J. Eur. Math. Soc. 10, 1 (2008).

[40] S.B. Kuksin, Hamiltonian PDEs, in Handbook of dynamical systems Eds. B. Hasselblatt and A. Katok, Elsvier, Amsterdam 1B, 1087 (2006).

[41] N. Navon, A.L. Gaunt, R.P. Smith and Z. Hadzibabic, Emergence of a turbulent cascade in a quantum gas, Nature 539, 72 (2016).

[42] R.S. Lous, I. Fritsche, M. Jag, B.Huang and R. Grimm, Thermometry of a deeply degenerated Fermi gas with a Bose-Einstein condensate, arXiv:1702.02772 condmat.quant-gas] (2017). 